Devi, P., Ponteconvo, G. \& Higginbottom, C. (1951). J. gen. Microbiol. 5, 781-787.

\title{
Mutations Affecting the Nutritional Requirements of Aerobacter aerogenes Induced by Irradiation of Dried Cells
}

\author{
By P. DEVI, G. PONTECoRvo and C. HigginbotToM \\ Department of Genetics, University of Glasgow \\ and the Hannah Dairy Research Institute, Kirkhill, Ayr
}

SUMMARY: By irradiating with X-rays Aerobacter aerogenes (AlOC) in the dry state, mutants have been obtained differing from the parent strain in specific growth-factor requirements. About half of the mutants differ from the parent, not in having an absolute requirement for a nutrilite, but in having a longer lag-phase without it.

The present paper deals with the isolation, after X-ray treatment of dried cells, of mutant strains of Aerobacter aerogenes which have growth-factor requirements additional to those of the parent strain (Beadle, 1945). The growth-factor requirements of most of the mutants were partially identified. Mutants were isolated which required one of the following substances, or substances metabolically related to them: aneurin, methionine, adenine, uracil, histidine, threonine, arginine, nicotinic acid, glycine, asparagine. The parent strain did not require any of these and grew well on a medium in which the only organic compound was glucose.

\section{EXPERIMENTAL}

Strain. The micro-organism used was a non-motile strain of $A$. aerogenes (AlOC) originally isolated by one of us (C. H.) from dried milk. It was purified by single cell isolation, and kept pure by occasional single colony isolations. It grew well on the defined medium (referred to as the 'minimal' medium) used by Lodge \& Hinshelwood (1943).

Media. The 'minimal' medium consisted of: $\left(\mathrm{NH}_{4}\right)_{2} \mathrm{SO}_{4}, 0.96 \mathrm{~g} . ; \mathrm{KH}_{2} \mathrm{PO}_{4}$, 3.46 g.; $\mathrm{MgSO}_{4} \cdot 7 \mathrm{H}_{2} \mathrm{O}, 0 \cdot 04 \mathrm{~g}$.; glucose, $20 \cdot 0 \mathrm{~g}$., all dissolved in $1 \mathrm{l}$. distilled water, the $\mathrm{pH}$ being adjusted to $7 \cdot 2$ with sodium hydroxide.

The 'complete' medium consisted of: peptone, $10.0 \mathrm{~g}$.; yeast extract (Yeastrel), 5.0 g.; glucose $10 \cdot 0$ g.; tap water, 1,000 ml.; pH 7-7.2. Both media were solidified, when necessary, with $1.5 \%$ of agar. For special purposes, both media were modified as mentioned below.

Irradiation. Irradiation was carried out at $7.5 \mathrm{~cm}$. from the tungsten target of an air-cooled Coolidge tube (Watson Mobilix X-ray unit with Aeromax 12 tube); peak KV 80, $5 \mathrm{~mA}$; output on the specimen about 1,500 roentgen/ min. The dose was varied by varying the time of exposure.

The drying of cells for irradiation. Cells grown $\left(24 \mathrm{hr}\right.$. at $\left.37^{\circ}\right)$ on agar slopes of complete medium were suspended in complete medium (diluted 1/1), centrifuged for $1 \mathrm{~min}$. at low speed to remove clumps, and the supernatant 
liquid adjusted to give a Spekker absorptiometer reading equivalent to a viable count of 12 to $14 \times 10^{7}$ cells $/ \mathrm{ml}$. Of this suspension $0.01 \mathrm{ml}$. was pipetted on to a sterile disk of Cellophane 600 (permeable) $1 \mathrm{~cm}$. in diameter; several such disks were placed in a sterile Petri dish and cooled for $1 \mathrm{hr}$. in the refrigerator. They were then transferred to a cooled vacuum desiccator over anhydrous calcium chloride and the pressure kept at $1 \mathrm{~mm}$. $\mathrm{Hg}$ for $2 \mathrm{hr}$. An average of $33 \%$ of the bacteria survived this treatment. When stored in a desiccator at atmospheric pressure and room temperature 50-70\% of the bacteria which had survived the drying on these disks were still alive after three weeks.

\section{Determination of nutritional requirements by auxanography}

A 24-hr. culture of a mutant in liquid complete medium was centrifuged, washed twice with sterile $\frac{1}{4}$ strength Ringer's solution, and a loopful of the third suspension spread on minimal medium agar plates and dried. A few minute crystals of each of the vitamins, amino-acids, purines and pyrimidines to be tested were put in marked positions on the inoculated agar surface and the plates incubated at $37^{\circ}$. As the parent culture would not grow on minimal medium alone, good confluent growth at any locus on the plate was taken to indicate that the mutant strain required as a nutrient that particular substance corresponding to the site of growth (Beijerinck, 1889; Pontecorvo, 1947, 1949). This was confirmed in liquid minimal medium to which the particular substance was added.

\section{Irradiation of dried cells}

Cellophane disks carrying a dried film of bacteria were placed in a small sterile Petri dish, the lid of which was made of Cellophane, and were irradiated with dosages of 135,000 or $180,000 \mathrm{r}$. administered in 90 and $120 \mathrm{~min}$. respectively. (By a mistake in calculation the dosages were stated as $\mathbf{3 5 , 0 0 0}$ and 50,000 r. in the preliminary report of Devi, Pontecorvo \& Higginbottom, 1947.) Several untreated disks were used as controls. The dried films of bacteria, from treated and control disks, were resuspended in $\frac{1}{4}$ strength sterile Ringer's solution, the suspension diluted to give 50-200 colonies/plate and plated. The technique of isolation was that of Lederberg \& Tatum (1946). With this technique presumptive mutant colonies are identified as delayed colonies which come up after the addition of a layer of complete medium, or one containing one or more growth factors, to plates initially prepared with minimal medium.

Both the complete and the minimal media given above had to be modified to check the vigorous gas production which otherwise cracked the agar in such layer plates. Glucose was entirely omitted from the complete medium and in the minimal medium the ammonium sulphate was increased to $5 \mathrm{~g}$./ $/$. and the glucose decreased to $5 \mathrm{~g}$. $/ \mathrm{l}$.

The suspension of cells (treated or control) was added to the middle layer of dishes plated with three layers (about $5 \mathrm{ml}$. each) of modified minimal medium. 
After incubation of the plates at $37^{\circ}$ for $36-38 \mathrm{hr}$. the colonies then visible were counted and marked on the back of the dish with spots of Indian ink. These colonies, having developed in minimal medium, were presumably produced by non-mutated bacteria. A fourth layer, of modified complete medium agar, was then added, and the plates incubated for a further $24 \mathrm{hr}$. A further small number of colonies usually developed and these were all isolated and tested. Only a few of these delayed colonies were found to be permanent mutants persistently unable to grow on minimal medium unless supplemented.

Table 1. The results of irradiating dried cells of Aerobacter aerogenes with $\boldsymbol{X}$-rays

\begin{tabular}{|c|c|c|c|c|}
\hline \multirow[b]{2}{*}{$\begin{array}{l}\text { Irradiation } \\
\text { dosage } \\
\text { (roentgens) }\end{array}$} & \multicolumn{2}{|c|}{$\begin{array}{l}\text { No. of colonies } \\
\text { which grew }\end{array}$} & \multirow[b]{2}{*}{$\begin{array}{c}\text { Confirmed } \\
\text { mutants } \\
\text { among } \\
\text { (b) }\end{array}$} & \multirow[b]{2}{*}{ Code number of each mutant } \\
\hline & $\begin{array}{c}\text { Before } \\
\text { addition } \\
\text { of } \\
\text { complete } \\
\text { medium } \\
\quad(a)\end{array}$ & $\begin{array}{l}\text { After } \\
\text { addition } \\
\text { of } \\
\text { complete } \\
\text { medium } \\
\text { (b) }\end{array}$ & & \\
\hline \multirow[t]{4}{*}{185,000} & 100 & 6 & 1 & 3A3 \\
\hline & 1220 & 27 & 5 & $4 \mathrm{A3}, 4,10^{+}, 15^{+}, 31$ \\
\hline & 122 & $\mathbf{2}$ & 1 & $\mathbf{5 A 2}$ \\
\hline & 1541 & 7 & $\mathbf{2}$ & $\mathrm{7A2}^{+}, \mathbf{4}^{+}$ \\
\hline \multirow{6}{*}{180,000} & 272 & 8 & 1 & $\mathbf{5 B 8}+$ \\
\hline & 735 & 15 & 4 & $7 \mathrm{B1}^{+}, \mathbf{2}^{+}, \mathbf{8}, 5^{+}$ \\
\hline & 1468 & 17 & 6 & $9 B 1,2^{+}, 4^{+}, 6^{+}, 16+, 17$ \\
\hline & 198 & 10 & $\mathbf{0}$ & \\
\hline & 1364 & 18 & $\mathbf{3}$ & $11 B 1,2+, 16$ \\
\hline & 1641 & 11 & 1 & $12 \mathrm{~B} 19$ \\
\hline \multirow[t]{2}{*}{ Total } & $\overline{8661}$ & $\overline{121}$ & $\overline{24}$ & \\
\hline & \multicolumn{2}{|c|}{ Non-irradiated controls } & & \\
\hline $\mathbf{0}$ & 181 & 0 & 0 & \\
\hline $\mathbf{0}$ & 555 & 10 & 1 & $\mathbf{5 C 1}$ \\
\hline 0 & 872 & 6 & 0 & \\
\hline 0 & 889 & 8 & $\mathbf{0}$ & \\
\hline 0 & 1809 & 4 & 0 & \\
\hline 0 & 2097 & 9 & 0 & \\
\hline 0 & 1728 & 17 & o & \\
\hline Total & $\overline{8131}$ & $\overline{\mathbf{5 4}}$ & $\overline{1}$ & \\
\hline
\end{tabular}

The results of a number of experiments (Table 1 ) show that of 8,185 colonies from non-irradiated dried bacteria only one was a mutant, while of 8,782 colonies from irradiated dried bacteria twenty-four were mutants. It is clear that irradiation increased the proportion of mutant colonies; but of the delayed colonies, which became visible only after the addition of complete medium, only a small proportion were mutants. Despite this, the stratification technique is certainly labour-saving; it involves the isolation of the delayed colonies only, among which the proportion of mutants in our experiments was 
about one in six. In preliminary experiments in which all colonies from irradiated cells plated on complete medium were isolated the proportion of mutants was usually well below one in a hundred.

An attempt to assess the survival rate after irradiation was made, but the results were too variable from experiment to experiment to allow a reliable estimate; in the ten series of irradiations tabulated in Table 1, the survival rate varied from $0 \cdot 1$ to $2 \cdot 0 / 1,000$. These rates were calculated by comparing, on complete medium, the viable counts of suspensions from non-irradiated disks of dried bacteria with the viable counts from an equal number of irradiated disks. Two main sources of variation were noted: $(a)$ the initial number of viable bacteria on each disk, $(b)$ the viable counts on the same suspension after irradiation according to the medium used for plating. No further attempt was made to standardize this part of the technique, and therefore no estimate could be made of mutation rates.

Table 2. The classification of 25 mutants according to growth-factor requirements

Growth-factor used
by the mutant
Nicotinic acid
Histidine
Uracil
Methionine
Serine or
Glycine
Threonine
Arginine
Adenine
Aneurin
Asparagine
Thiosulphate
Yeast extract

Unknown (strain
lost)

\begin{tabular}{|c|c|c|}
\hline \multirow{2}{*}{$\begin{array}{l}\text { No. of } \\
\text { mutants }\end{array}$} & \multicolumn{2}{|c|}{ Mutant No. } \\
\hline & Non-adaptable & Adaptable \\
\hline $\mathbf{1}$ & 3A3 & - \\
\hline 1 & . & 9B2 \\
\hline 2 & $5 \mathrm{Cl}$ & $4 A 10$ \\
\hline 1 & 4A4 & . \\
\hline 1 & $7 \mathrm{B3}$ & . \\
\hline 1 & 9B1 & - \\
\hline 1 & $12 B 19$ & . \\
\hline 1 & 4A31 & - \\
\hline 3 & $11 B 16$ & 4A15, 7A2 \\
\hline 1 & - & $11 \mathrm{B2}$ \\
\hline 2 & 4A3 & 7A4 \\
\hline 9 & 5A2, 9B17 & $\begin{array}{l}7 \mathrm{B1}, 7 \mathrm{B2} \\
7 \mathrm{B5}, \mathbf{5 B 8}, \\
9 \mathrm{~B} 4,9 \mathrm{B6}, \\
9 \mathrm{B16}\end{array}$ \\
\hline 1 & 11B1 & - \\
\hline
\end{tabular}

\section{Types of mutants obtained}

In all, 25 mutants were obtained, of which one was from non-irradiated bacteria and 24, from irradiated bacteria. As shown in Table 2, for 15 of the 25 mutants it was possible to identify one or more substances which allowed growth to occur when added as a supplement to the minimal medium. Of the remaining 10 strains, one (11B1) was lost and for the others attempts to find the additional substance or substances required for growth were unsuccessful. Failure may indicate either a multiple requirement or a requirement for substances other than those included in our tests.

The mutants obtained were of two main types; all required for immediate growth the addition to the minimal medium of a particular compound or a 
complex nutrient mixture such as yeast extract. Some, however, eventually grew even without this addition after a lag of $24 \mathrm{hr}$. or more; these are described here as 'adaptable'. Thirteen of the 25 mutants were of the adaptable type. Preliminary experiments on a number of these adaptable mutants revealed the complexity of the process of adaptation, which has been emphasized by all careful workers. Taken at their face value our results are compatible with a mechanism of reversible mutation from growth-factor dependence to independence and vice versa, coupled with differential growth rates such as to lead to an equilibrium between dependent and independent cells, the position of equilibrium varying with the medium. The work of Ryan $(1946,1948)$ has shown how delicately adjustable a mechanism like this may be. However, other possibilities cannot be ruled out, but since our experiments on this aspect of the work were not conclusive we refrain from reporting them here.

Table 3. Alternative growth-factors tested with 13 mutants

Growthfactor first identified

Nicotinic acid

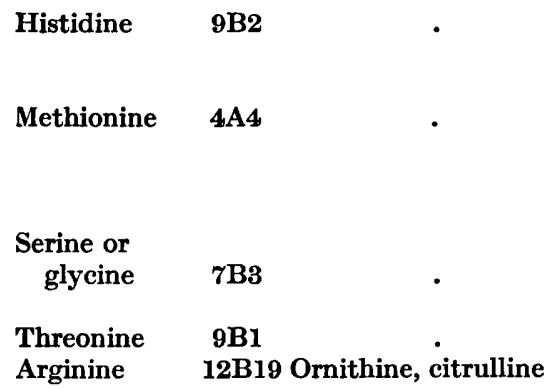

Adenine

Aneurin

Aneurin

Thiosulphate

Thiosulphate Uracil GM V 4

\section{Strain (a) effective}

3A3 Nicotinamide guanylic acid

$11 B 16$ Vitamin thiazole formamide

4A3 As 7A4, sulphite
Other substances tested and found to be

4A31 Adenosine, adenylic acid, guanine, guanosine,

4A15 Vitamin thiazole, vita-

7A2 $\}$ min pyrimidine, thio-

7A4 Metabisulphite, hyposulphite, methionine, cystine, cysteine, thioformamide, thiourea, thiolacetic acid

5C1 Cytidine, cytidylic acid

\section{(b) ineffective}

Anthranilic acid, indole, tryptophan, quinolinic acid, picolinic acid, pyridine, piperidine, ornithine, proline, $\alpha$-amino- $n$-valeric acid, trigonelline, kynurenic acid, 3hydroxy-anthranilic acid.

4-Hydroxy-methyl glyoxaline alone or plus alanine or glycine, anserine, benziminazole.

Choline, cystine, cysteine, thioformamide, cystine plus choline, taurine, thiolacetic acid, thiodiglycol, sulphanilic acid, phenylthiourea.

Alanine, $\beta$-alanine, pyruvic acid, propionic acid, acetate.

$\alpha$-Aminobutyric acid, sodium butyrate.

Glutamic acid, proline, guanidine and $\alpha$-amino- $n$-valeric acid (singly and together), ammonium carbamate, urea, creatinine.

Cytidine, uracil, thymine, uric acid, allantoin, 2, 4, 5,-triamino-6-hydroxy-pyrimidine, benziminazole.

Thioformamide, vitamin pyrimidine.

Persulphate, sulphite, sulphate.

Persulphate, sulphate.

Thymine, uric acid, allantoin. 
The information on the growth responses of the mutants is summarized in Table 3. Of the 15 mutants for which a necessary growth-factor was identified 13 were further tested for their ability to grow with alternative substances. Unfortunately, not many of the substances which should have been tested were available at the time this work was done. However, from inspection of Table 3, the following remarks can be made.

Reduction of sulphate. The two strains 7A4 and 4A3 suggest two different blocks in the reduction of sulphate; 7A4 was unable to utilize sulphite but grew on more reduced compounds, while $4 \mathrm{A3}$ was able to utilize it (cf. Fries, 1946, for Ophiostoma; Hockenhull, 1948, for Aspergillus).

Biosynthesis of nicotinic acid. For strain 3A3 anthranilic acid, 3-hydroxyanthranilic acid, indole, tryptophan and kynurenic acid were unable to replace nicotinic acid. The block in the biosynthesis of nicotinic acid in this mutant may occur at some late stage in the synthesis of nicotinic acid as with some of the mutants of Neurospora described by Yanofsky \& Bonner (1950).

Biosynthesis of adenine. The mutant 4A31 could utilize guanine instead of adenine, a property which it shares with some mutants of $B$. subtilis (Burkholder \& Giles, 1947) but not with the numerous mutants of Ophiostoma (Fries, 1946), Neurospora (Mitchell \& Houlahan, 1946) or Aspergillus nidulans (Pontecorvo, unpubl.)

Biosynthesis of aneurin. The non-adaptable mutant $11 \mathrm{B16}$ could utilize the thiazole moiety of aneurin, but not the pyrimidine moiety; it could not use thioformamide in place of the thiazole. The lag-phase of the two adaptable mutants 4A15 and 7A2 was shortened by the presence of either moiety of aneurin or by thioformamide.

\section{Conclusions}

The present work has shown that bacterial cells irradiated in the dry state can give rise to nutritional mutants. Taken together with the results of Burkholder \& Giles (1947) on the irradiation of bacterial spores, this confirms that active growth and division at the time of irradiation are not essential conditions for mutation induced by $\mathbf{X}$-irradiation. About half of the mutants obtained differed from the parent strain not by an absolute dependence on specific nutrient but in showing an extended lag in the absence of it. The mechanisms of this extended lag might be of the kind suggested by Hinshelwood (1946), of the kind usually referred to as mutation-plus-selection, or of other types, as suggested by the work of Sonneborn (1946) with Paramecium and of Ephrussi, Hottinguer \& Tavlitzki (1949) with yeast.

A scholarship from the Government of India has enabled one of us (P.D.) to participate in this investigation. The authors wish to acknowledge gifts of chemicals from Professor H. A. Krebs, Drs W. V. Thorpe, E. R. Dawson, H. McIlwain and E. C. Bate-Smith, and Roche Products Ltd. 


\section{REFERENCES}

Beadle, G. W. (1945). Biochemical Genetics. Chem. Rev. 37, 15.

Beijerinck, M. W. (1889). L'auxanographie, ou la méthode de l'hydrodiffusion dans la gelatine appliques aux recherches microbiologiques. Arch. néerl. Sci. 23,367 .

Burkholder, P. R. \& Gires, N. (1947). Induced biochemical mutations in Bacillus subtilis. Amer. J. Bot. 34, 345.

Devi, P., Ponteconvo, G. \& Higginbottom, C. (1947). X-ray induced mutations in dried bacteria. Nature, Lond., 160, 503.

Ephrussi, B., Hottinguer, H. \& TAvlitzki, J. (1949). Action de l'acriflavine sur les levures. Ann. Inst. Pasteur. 76, 419.

FrIEs, N. (1946). Mutant strains of Ophiostoma multiannulatum requiring components of different nucleotides. Ark. Bot. 33A, 1.

Hinshelwood, C. N. (1946). The chemical kinetics of the bacterial cell. Oxford: University Press.

Hockenhuld, D. (1948). Mustard-gas mutation in Aspergillus nidulans. Nature, Lond., 161, 100.

Lederberg, J. \& Tatum, E. L. (1946). Detection of biochemical mutants of microorganisms. J. biol. Chem. 165, 381.

Lodge, R. M. \& Hinshelwood, C. N. (1943). Physicochemical aspects of bacterial growth. Part VIII. Growth of Bact. lactis aerogenes in media containing ammonium sulphate or various amino-acids. J. chem. Soc. 208.

Mitchell, H. K. \& Houlahan, M. B. (1946). Adenine-requiring mutants of Neurospora crassa. Fed. Proc. 5, 370.

Ponteconvo, G. (1947). The genetical control of nutritional requirements in microorganisms and its application to microbiological assays. Proc. Nutr. Soc. 5, 182.

Ponteconvo, G. (1949). Auxanographic techniques in biochemical genetics. J. gen. Microbiol. 3, 122.

RYan, F. J. (1946). Back mutation and adaptation of nutritional mutants. Cold Spr. Harb. Sym. quant. Biol. $11,215$.

Ryan, F. J. (1948). On the stability of nutritional mutants of bacteria. Proc. nat. Acad. Sci., Wash. 34, 426.

Sonneborn, T. M. (1946). Experimental control of the concentration of cytoplasmic genetic factors in Paramecium. Cold. Spr. Harb. Sym. quant. Biol. 11, 236.

YANOFSKY, C. \& BonNer, D. M. (1950). Evidence for the participation of kynurenine as a normal intermediate in the biosynthesis of niacin in Neurospora. Proc. nat. Acad. Sci., Wash. 36, 167. 